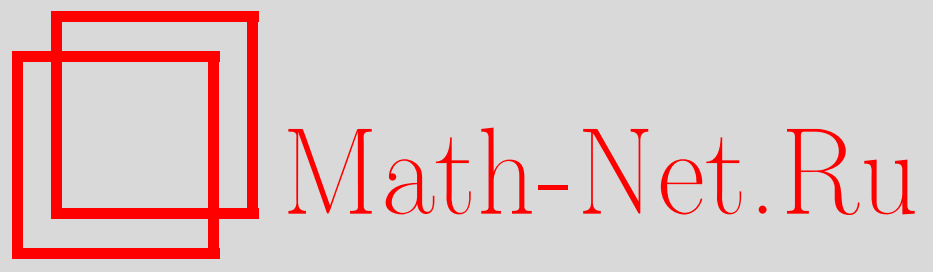

Обцероссийский математический портал

Н. О. Седова, Вырожденные функции в исследовании асимптотической устойчивости решений функциональнодифференциальных уравнений, Матем. заметки, 2005, том 78, выпуск 3, 468-472

DOI: https://doi.org/10.4213/mzm2603

Использование Общероссийского математического портала Math-Net.Ru подразумевает, что вы прочитали и согласны с пользовательским соглашением http://www.mathnet.ru/rus/agreement

Параметры загрузки:

IP: 18.234 .197 .8

26 апреля 2023 г., 13:09:13 


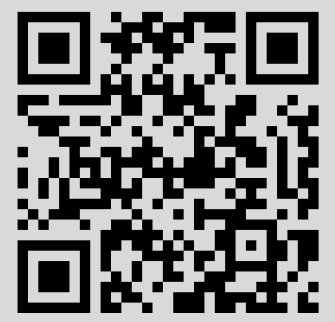




\section{ВЫРОЖДЕННЫЕ ФУНКЦИИ В ИССЛЕДОВАНИИ АСИМПТОТИЧЕСКОЙ УСТОЙЧИВОСТИ РЕШЕНИЙ ФУНКЦИОНАЛЬНО-ДИФФЕРЕНЦИАЛЬНЫХ УРАВНЕНИЙ}

\section{Н. О. Седова}

Основным прямым методом исследования устойчивости для дифференциальньх уравнений является, как известно, метод Ляпунова. Отсутствие в общем случае эффективных алгоритмов построения функции с заданньми свойствами для конкретной задачи определило одно из главных направлений развития этого метода - поиск возможностей расширения класса вспомогательных функций. Для функционально-дифференциальных уравнений с запаздыванием прямой метод развивается в двух направлениях. Первое - метод функционалов Ляпунова-Красовского. Развитие классических теорем этого метода представлено, например, в [1]-[4].

В настоящей работе исследование проводится в рамках другого направления - метода функций, основы которого были заложены Б. С. Разумихиньм (см. [5]). Предлагается исследовать устойчивость и асимптотическую устойчивость нулевого решения неавтономного уравнения с конечным запаздыванием, используя так называемые вырожденные вспомогательные функции. Отличие их от классических функций Ляпунова в том, что они не обязательно положительно определены, а лишь неотрицательны. Кроме того, в теоремах об асимптотической устойчивости допускается, что производная такой функции также может быть лишь знакопостоянной. Полученные теоремы развивают, в частности, результаты работ [6]-[11].

В работе используются следующие обозначения: $\mathbb{R}^{n}$-пространство действительных $n$-векторов с нормой $|\cdot|, \mathbb{R}^{+}=[0,+\infty), C(X, Y)$ - пространство непрерьвных отображений $X \rightarrow Y$. Определим для $r>0$ пространство $C:=C\left([-r, 0], \mathbb{R}^{n}\right)$ функций $\varphi$ с нормой $\|\varphi\|=\max \{|\varphi(s)|:-r \leqslant$ $s \leqslant 0\}$ и множества $C_{a}=\{\varphi \in C:\|\varphi\|<a\}, \bar{C}_{a}=\{\varphi \in C:\|\varphi\| \leqslant a\}$ для произвольного $a>0$.

Пусть $\alpha \in \mathbb{R}^{+}$и $\beta>0$. Для непрерьвной функции $x:[\alpha-r, \alpha+\beta) \rightarrow \mathbb{R}^{n}$ и $t \in[\alpha, \alpha+\beta)$ определим $x_{t} \in C$ по формуле $x_{t}(s)=x(t+s),-r \leqslant s \leqslant 0$.

Рассмотрим функционально-дифференциальное уравнение

$$
\dot{x}(t)=X\left(t, x_{t}\right), \quad X(t, 0)=0,
$$

где $X \in C\left(\mathbb{R}^{+} \times C_{H}, \mathbb{R}^{n}\right), 0<H \leqslant+\infty$ и для каждого числа $q \in(0, H)$ существует неубьвающая функция $\mu_{q}(u), \mu_{q}(0)=0$ такая, что $\left|\int_{t_{1}}^{t_{2}} X(\tau, u(\tau)) d \tau\right| \leqslant \mu_{q}\left(\left|t_{2}-t_{1}\right|\right)$ для любого непрерьвного отображения $u:[a, b] \rightarrow \bar{C}_{q}$ и любых $t_{1}, t_{2} \in[a, b]$. Тогда для каждой начальной точки $(\alpha, \varphi) \in \mathbb{R}^{+} \times C_{H}$ существует непродолжаемое решение $x(t ; \alpha, \varphi)$ уравнения (1), определенное для $t \in[\alpha-r, \beta)$ и такое, что $x_{\alpha}(\alpha, \varphi)=\varphi[4]$. Кроме того, функционал $X(t, \varphi)$ удовлетворяет

Работа выполнена при финансовой поддержке Российского фонда фундаментальных исследований, грант № 02-01-00877. 
условию Липшица: для каждого множества $\bar{C}_{q}, q<H$, существует $l=l(q)>0$ такое, что для всех $\varphi_{1}, \varphi_{2} \in \bar{C}_{q}$ выполняется неравенство $\left|X\left(t, \varphi_{2}\right)-X\left(t, \varphi_{1}\right)\right| \leqslant l\left\|\varphi_{2}-\varphi_{1}\right\|$.

Дополнительно нам понадобятся следующие предположения и построения.

ПРЕДПОЛОЖЕнИЕ 1. Функчионал $X(t, \varphi)$ ограничен и равномерно непрерывен на каждом множестве $\mathbb{R}^{+} \times K$, где $K \subset C_{H}-$ компакт.

Данному предположению удовлетворяют, в частности, все непрерывные функционалы вида $X(\varphi)$, а также периодические и почти периодические по $t$.

Обозначим $\mathscr{F}_{X}=C\left(\mathbb{R}^{+} \times C_{H}, \mathbb{R}^{n}\right)$, и пусть $X^{\tau} \in \mathscr{F}_{X}$ - сдвиг функционала $X$, определяемый равенством $X^{\tau}(t, \varphi)=X(\tau+t, \varphi)$. Определим сходимость в $\mathscr{F}_{X}$ как равномерную на каждом компакте $K^{\prime} \subset \mathbb{R}^{+} \times C_{H}$. Тогда семейство $\left\{X_{\tau}(t, \varphi)=X(\tau+t, \varphi), \tau \in \mathbb{R}^{+}\right\}$предкомпактно в $\mathscr{F}_{X}$ и уравнению (1) можно сопоставить семейство предельных уравнений $[12] \dot{x}(t)=X^{*}\left(t, x_{t}\right)$, где $X^{*}(t, \varphi) \in \mathscr{F}_{X}$ есть предельный к $X$ функционал, определенньй равенством

$$
\lim _{t_{k} \rightarrow+\infty} X\left(t_{k}+t, \varphi\right)=X^{*}(t, \varphi)
$$

В силу условия Липшица решение уравнения (1) и каждого предельного к нему уравнения для каждой точки $(\alpha, \varphi) \in \mathbb{R}^{+} \times C_{H}$ будет единственньм.

Кроме стандартных определений устойчивости нулевого решения по Ляпунову для уравнения (1) (см., например, [12]), мы используем следующие

ОПРЕДЕЛЕниЕ 1. Нулевое решение уравнения (1) называется равномерно притяљивающим относительно множества $\Lambda \subset C_{H}$, если существует $\Delta>0$ и для любого $\varepsilon>0$ существует $T=T(\varepsilon)>0$ такое, что для любой начальной точки $(\alpha, \varphi) \in \mathbb{R}^{+} \times\left[C_{\Delta} \cap \Lambda\right]$ справедливо неравенство $|x(t ; \alpha, \varphi)|<\varepsilon$, как только $t>\alpha+T$. Нулевое решение уравнения (1) назьвается (равномерно) асимптотически устойчивым относительно множества $\Lambda \subset C_{H}$, если условия (равномерной) асимптотической устойчивости выполняются для $\varphi \in \Lambda$.

ОПРЕДЕЛЕНИЕ 2. Нулевое решение назьвается асимптотически устойчивым относительно множества $\Lambda \subset C_{H}$ равномерно по $\left\{\dot{x}(t)=X^{*}\left(t, x_{t}\right)\right\}$, если для любого $\varepsilon>0$ и любого $\alpha \in \mathbb{R}$ можно указать $\delta=\delta(\varepsilon, \alpha)>0$ и $\Delta=\Delta(\alpha)>0$ такие, что из $\varphi \in \Lambda \cap\{\|\varphi\| \leqslant \delta\}$ следует $\left|x^{*}(t ; \alpha, \varphi)\right| \leqslant \varepsilon$ при всех $t \geqslant \alpha$, а из $\varphi \in \Lambda \cap\{\|\varphi\| \leqslant \Delta\}$ следует $\left|x^{*}(t ; \alpha, \varphi)\right| \rightarrow 0$ при $t \rightarrow+\infty$ для каждого решения $x^{*}(t ; \alpha, \varphi)$ любого предельного уравнения $\dot{x}(t)=X^{*}\left(t, x_{t}\right)$.

Функцию $V \in C^{1}\left(\mathbb{R}^{+} \times G_{H}, \mathbb{R}^{+}\right)$, где $G_{H}=\left\{x \in \mathbb{R}^{n}:|x|<H\right\}$, удовлетворяющую условию $V(t, 0) \equiv 0$, назовем функиией Ляпунова. Ее производная в силу уравнения (1) есть функционал $V^{\prime}: \mathbb{R}^{+} \times C_{H} \rightarrow \mathbb{R}$, определяемьй формулой

$$
V^{\prime}(t, \varphi)=\frac{\partial V(t, \varphi(0))}{\partial t}+\sum_{i=1}^{n} \frac{\partial V(t, \varphi(0))}{\partial x_{i}} X_{i}(t, \varphi) .
$$

Обозначим $\Omega_{t}(V)=\left\{\varphi \in C_{H}: V(t+s, \varphi(s)) \leqslant V(t, \varphi(0)),-r \leqslant s \leqslant 0\right\}$. Во всех приведенных ниже утверждениях мы дополнительно предполагаем, что $V^{\prime}(t, \varphi) \leqslant 0$ для всех $t \in \mathbb{R}^{+}$и $\varphi \in \Omega_{t}(V)$.

Рассмотрим задачу об исследовании устойчивости нулевого решения уравнения (1). Пусть сначала правая часть уравнения удовлетворяет дополнительному предположению 1 . Классическая теорема Разумихина [5] утверждает, что для устойчивости достаточно существование положительно определенной функции Ллпунова, производная которой в силу уравнения неположительна для всех $(t, \varphi): t \in \mathbb{R}^{+}, \varphi \in \Omega_{t}(V)$. Оказывается, использование определенных свойств предельных уравнений позволяет применять для доказательства устойчивости знакопостоянные функции. Для каждого числа $c \in \mathbb{R}^{+}$и непрерьвной функции $V(t, x)$ определим множество

$$
\begin{aligned}
V_{\max }^{-1}(\infty, c)= & \left\{\varphi \in C_{H} \mid \exists \varphi_{n} \rightarrow \varphi, t_{n} \rightarrow+\infty:\right. \\
& \left.\lim _{n \rightarrow \infty} \max _{-r \leqslant s \leqslant 0} V\left(t_{n}+s, \varphi_{n}(s)\right)=\lim _{n \rightarrow \infty} V\left(t_{n}, \varphi_{n}(0)\right)=c\right\} .
\end{aligned}
$$


Определим также класс функций $\mathscr{K}=\left\{\sigma \in C\left(\mathbb{R}^{+}, \mathbb{R}^{+}\right), \sigma(u)\right.$ строго возрастает и $\left.\sigma(0)=0\right\}$.

Тогда если существует функция Ляпунова $V$ такая, что нулевое решение асимптотически устойчиво относительно множества $V_{\max }^{-1}(\infty, 0)$ равномерно по $\left\{\dot{x}(t)=X^{*}\left(t, x_{t}\right)\right\}$, то нулевое решение уравнения (1) устойчиво. Если при этом $V(t, x) \leqslant b(|x|), b \in \mathscr{K}$, то нулевое решение уравнения (1) равномерно устойчиво [11].

Для исследования асимптотической устойчивости нам потребуются следующие дополнительные предположения [8].

ПРЕДПОЛОЖЕНИЕ 2. Функиия $V(t, x)$ равномерно непрерывна и ограничена на множесmвах вида $\mathbb{R}^{+} \times \bar{G}_{q}$, әде $\bar{G}_{q}=\left\{x \in \mathbb{R}^{n}:|x| \leqslant q\right\}, 0<q<H$.

ПРЕДПОЛОЖЕНИЕ 3. ФУнкиионал $U(t, \varphi)=V^{\prime}(t, \varphi)$ равномерно непрерывен и ограничен на мнохествах вида $\mathbb{R}^{+} \times K, K \subset C_{H}-$ жомпакт.

При таких условиях семейства сдвигов $\left\{V_{\tau}(t, x), \tau \in \mathbb{R}^{+}\right\}$и $\left\{U_{\tau}(t, \varphi), \tau \in \mathbb{R}^{+}\right\}$предкомпактны в соответствующих пространствах $\mathscr{F}_{V}$ и $\mathscr{F}_{U}$ с метризуемой компактно-открытой топологией [12], [13] и существуют предельные функции, любую из которых мы будем обозначать $V^{*}(t, x)$, и предельные функционалы $U^{*}(t, \varphi)$.

Теперь для произвольных $c_{0}, t \in \mathbb{R}^{+}$и последовательности $t_{n} \rightarrow+\infty$, определяющей предельные $V^{*}$ и $U^{*}$, обозначим

$$
M\left(t, V^{*}, c_{0}\right)=\left\{\varphi \in C_{H} \mid \max _{-r \leqslant s \leqslant 0} V^{*}(t+s, \varphi(s))=V^{*}(t, \varphi(0))=c_{0}\right\}
$$

$L\left(t, U^{*}\right)=\left\{\varphi \in C_{H} \mid U^{*}(t, \varphi)=0\right\}$. Вместо множества $V_{\max }^{-1}(\infty, 0)$ будем использовать

$$
N=\left\{\varphi \in C_{H} \mid \exists V^{*}(t, x): V^{*}(s, \varphi(s))=0, s \in[-r, 0]\right\}
$$

(для функции Ляпунова $V$, удовлетворяющей предположению $2, N \equiv V_{\max }^{-1}(\infty, 0)$ ).

Теорема Красовского об асимптотической устойчивости [14] использует положительно определенную функцию, производная которой отрицательно определена для каждого $t \in \mathbb{R}^{+}$на множестве $\Omega_{t}(V, \eta)=\left\{\varphi \in C_{H}: V(t+s, \varphi(s)) \leqslant \eta(V(t, \varphi(0))),-r \leqslant s \leqslant 0\right\}$, где функция $\eta \in \mathscr{K}$, $\eta(u)>u$ при $u>0$ (очевидно, $\Omega_{t}(V, \eta)$ содержит $\Omega_{t}(V)$, но в общем случае не совпадает с ним).

Для уравнений с правой частью, удовлетворяющей дополнительному предположению 1 , в работе [8] асимптотическая устойчивость доказана в предположении существования знакоопределенной функции Ляпунова с производной, знакопостоянной на множестве $\Omega_{t}(V)$. Однако и этот результат можно улучшить, отказавшись от знакоопределенности функции $V$.

А именно, если правая часть уравнения (1) удовлетворяет предположению 1 , то в приведенных обозначениях справедлив следующий результат [11].

Теорема 1. Предположим, что существует функиия Ляпунова $V: \mathbb{R}^{+} \times G_{H} \rightarrow \mathbb{R}^{+}$ такая, что выполняются предположения 2-3, нулевое решение асимптотически устойчиво относительно мнохсества $N$ равномерно по $\left\{\dot{x}(t)=X^{*}(t, \varphi)\right\}$ и для любого достаточно малого $c>0$ существует последовательность $t_{n} \rightarrow+\infty$, для которой мнохество $M\left(t, V^{*}, c\right) \cap L\left(t, U^{*}\right)$ не содерхит решений уравнения $\dot{x}=X^{*}\left(t, x_{t}\right)$. Тогда нулевое решение уравнения (1) эквиасимптотически устойчиво. Если вдобавок $V(t, x) \leqslant b(|x|)$, $b \in \mathscr{K}$, и последнее условие выполняется для любой последовательности $t_{n} \rightarrow+\infty$, то нулевое решение уравнения (1) равномерно асимптотически устойчиво.

Для случая, когда правая часть уравнения не зависит от $t$ или периодична по $t$ c некоторым периодом $T$, из приведенной теоремы легко получить следствия, в которых не используются предельные уравнения, поскольку для $X(t, \varphi) \equiv X(\varphi)$ все предельные функционалы совпадают с $X(\varphi)$, а для периодического $X(t, \varphi)$ имеют вид $X^{*}(t, \varphi)=X(t+\gamma, \varphi), \gamma \in[0, T)$. Отсюдаполучаем следующие утверждения.

Теорема 2. Пусть $X(t, \varphi) \equiv X(\varphi)$ и существует функция Ляпунова $V: G_{H} \rightarrow \mathbb{R}^{+}$ такая, что нулевое решение асимптотически устойчиво относительно мнохсества 
$\left\{\varphi \in C_{H}: V(\varphi(s)) \equiv 0, s \in[-r, 0]\right\}$. Тогда нулевое решение уравнения (1) равномерно устойчиво. Если при этом мнохсество

$$
\left\{\varphi \in C_{H}: \max _{-r \leqslant s \leqslant 0} V(\varphi(s))=V(\varphi(0))>0, V^{\prime}(\varphi)=0\right\}
$$

не содержит решений уравнения (1), то нулевое решение уравнения (1) равномерно асимптотически устойчиво.

Tеорема 3. Пусть для некоторого $T>0 \quad X(t, \varphi) \equiv X(t+T, \varphi)$ и существует функиия Ляпунова $V: \mathbb{R}^{+} \times G_{H} \rightarrow R^{+}$такая, что $V(t+T, x) \equiv V(t, x)$ и нулевое решение асимптотически устойчиво относительно мнохсества $\left\{\varphi \in C_{H}: V(s, \varphi(s)) \equiv 0, s \in[-r, 0]\right\}$. Тогда нулевое решение уравнения (1) равномерно устойчиво. Если при этом множествo

$$
\left\{\varphi \in C_{H}: \max _{-r \leqslant s \leqslant 0} V(t+s, \varphi(s))=V(t, \varphi(0))>0, V^{\prime}(t, \varphi)=0\right\}
$$

не содержит решений уравнения (1), то нулевое решение уравнения (1) равномерно асимптотически устойчиво.

Теорема 2 обобщает теорему из [7] на случай знакопостоянной функции $V(x)$; теорема 3 развивает аналогичный результат из [9], полученный в предположении существования положительно определенной функции $V(t, x)$ с неположительной на множестве $\Omega_{t}(V, \eta)$ производной.

Пусть теперь правая часть уравнения (1) является равномерно почти периодической по $t$, т.е. для любого $\varepsilon>0$ и каждого компакта $K \subset C_{H}$ существует число $L=L(\varepsilon, K)>0$ такое, что для любого $a \in \mathbb{R}$ найдется $\tau \in[a, a+L]$, при котором для всех $t \in \mathbb{R}, \varphi \in K$ выполняется неравенство $|X(t+\tau, \varphi)-X(t, \varphi)|<\varepsilon$. Тогда функционал $X(t, \varphi)$ удовлетворяет предположению 1 и существует предельный функционал $X^{*}(t, \varphi) \equiv X(t, \varphi)$. Поэтому из теоремы 1 следует

Tеорема 4. Пусть $X(t, \varphi)$ - равномерно почти периодический по $t$ и сущ,ствует функиия Ляпунова $V: G_{H} \rightarrow \mathbb{R}^{+}$такая, что выполняется предполохение 3 , нулевое решение уравнения (1) равномерно асимптотически устойчиво относительно $\left\{\varphi \in C_{H}\right.$ : $V(\varphi(s)) \equiv 0, s \in[-r, 0]\}$, а мнохсество

$$
\left\{\varphi \in C_{H}: \max _{-r \leqslant s \leqslant 0} V(\varphi(s))=V(\varphi(0))>0, V^{\prime}(t, \varphi)=0\right\}
$$

не содержит решений уравнения (1). Тогда нулевое решение уравнения (1) эквиасимптотически устойчиво.

Приведем теперь результаты об устойчивости и асимптотической устойчивости для уравнения (1), правая часть которого не удовлетворяет предположению 1 . Помимо множества $V_{\max }^{-1}(\infty, c)$ используем также множество

$$
U^{-1}(\infty, 0)=\left\{\varphi \in C_{H} \mid \exists \varphi_{n} \rightarrow \varphi, t_{n} \rightarrow+\infty: \lim _{n \rightarrow \infty} U\left(t_{n}, \varphi_{n}\right)=0\right\},
$$

определяемое функционалом $U: \mathbb{R}^{+} \times C_{H} \rightarrow \mathbb{R}$.

Tеорема 5. Если существует функция Ляпунова $V: \mathbb{R}^{+} \times G_{H} \rightarrow \mathbb{R}^{+}$такая, ито нулевое решение является равномерно притягиваюшим относительно мнохества $V_{\max }^{-1}(\infty$, $0)$, то нулевое решение уравнения (1) устойчиво. Если к тому же $V(t, x) \leqslant b(|x|)$ для некоторой функции $b \in \mathscr{K}$, то нулевое решение уравнения (1) равномерно устойчиво. Наконеи, потребовав дополнительно, чтобъ $\left|V^{\prime}(t, \varphi)\right| \geqslant U(t, \varphi) \geqslant 0$ для всех $(t, \varphi) \in \mathbb{R}^{+} \times C_{H}$ (әде $U(t, \varphi)$ - функционал, равномерно непрерывный на каждом множестве $\mathbb{R}^{+} \times K$, $K \subset C_{H}$ - компакт), и чтобы множсество $U^{-1}(\infty, 0) \cap V_{\max }^{-1}(\infty, c)$ было пусто при с $>0$, получим достаточные условия для равномерной асимптотической устойчивости нулевого решения уравнения (1). 
Если на множестве $\left\{(t, \varphi) \in \mathbb{R}^{+} \times C_{H}: \varphi \in \Omega_{t}(V)\right\}$ справедлива оценка $V^{\prime}(t, \varphi) \leqslant-c(V(t$, $\varphi(0))), c \in \mathscr{K}$, то, очевидно, последнее условие теоремы 5 выполняется (аналогичная оценка предложена в [10] при исследовании асимптотической устойчивости решения обыкновенного дифференциального уравнения). В случае предкомпактности правой части уравнения (1) теорема 5 является следствием теоремы 1 , а в случае автономной или периодической правой части совпадает соответственно с теоремами 2 и 3 . Из теоремы 5 следует, что если в последнем условии теоремы 4 равенство $V^{\prime}(t, \varphi)=0$ заменить соотношением $\varphi \in U^{-1}(\infty, 0)$, то получим достаточные условия равномерной асимптотической устойчивости нулевого решения уравнения (1) с равномерно почти периодической по $t$ правой частью (заметим, что в отличие от автономного и периодического случая, для почти периодического функционала $X(t, \varphi)$ асимптотическая устойчивость не обязана быть равномерной, более того, нулевое решение такого уравнения может быть равномерно устойчивьм и притягивающим, но не являться равномерно асимптотически устойчивым).

\section{СПИСОК ЦИТИРОВАННОЙ ЛИТЕРАТУРЫ}

1. Андреев А. С., Павликов С. В. // ПММ. 1999. Т. 63. №1. С. 3-12. 2. Андреев А. С., Хусанов Д.Х. // Дифференц. уравнения. 1998. Т. 34. №7. С. 876-885. 3. Колмановский В.Б., Носов В.Р. Устойчивость и периодические режимы регулируемых систем с последействием. М.: Наука, 1981. 4. Хейл Дж. Теория функционально-дифференциальных уравнений. М.: Мир, 1984. 5. Разумихин Б. С. // ПММ. 1956. Т. 20. №4. С. 500-512. 6. Андреев А. С. // Докл. РАН. 1997. Т. 356. № 2. C. 151-153. 7. Haddock J., Terj'eki J. // J. Differential Equations. 1983. V. 48. P. 95-122. 8. Andreev A. S., Sedova N. O. // Functional Differential Equations. 1998. V. 5. P. 21-37. 9. Ким А. В. $i$-Гладкий анализ и функционально-дифференциальные уравнения. Екатеринбург: УрО РАН, 1996. 10. Iggidr A., Sallet G. // Automatica. 2003. V. 39. P. 167-171. 11. Sedova N. // J. Math. Anal. Appl. 2003. V. 281. №1. P. 307-319. 12. Андреев А. С., Хусанов Д. Х. // Дифференц. уравнения. 1998. Т. 34. №4. C. 435-440. 13. Sell G. R. // Trans. Amer. Math. Soc. 1967. V. 127. P. 214-262. 14. Красовский Н. Н. // ПММ. 1956. Т. 20. № 4. C. 513-518.

Ульяновский государственный университет

E-mail: SedovaNO@ulsu.ru 